\section{BLOT OUT THE LIEHT}

Seeing an Earth-like planet orbiting a star 10 billion times brighter has been likened to trying to see a firefly next to a lantern from 10 kilometres away. To block the starlight, astronomers are developing five types of coronagraph:

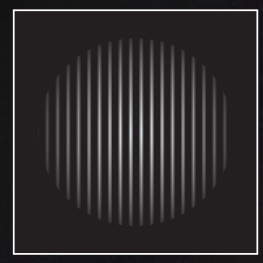

BAND LIMITED

Using a mask that resembles a barcode, this

coronagraph suppresses starlight along the telescope's optical axis while transmitting light from off-axis planets.

Key challenge:

Precision manufacture of the barcode-like mask

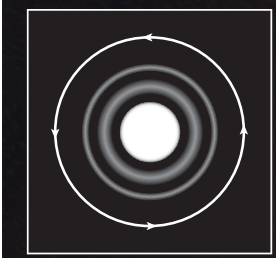

\section{VECTOR VORTEX}

A circular mask rotates the angle of polarization of photons from the star, so they cancel each other out.

Key challenge:

The need to manufacture the fine circular pattern of the mask, made from liquid-crystal polymers, to a precision of micrometres.

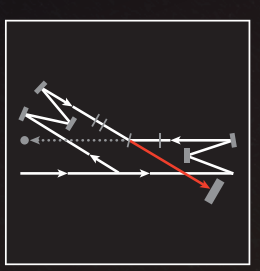

VISIBLE NULLING

Akin to flying several telescopes in formation, a device installed in a single spacecraft generates an interference pattern that suppresses starlight.

Key challenge:

Extending the technique to a broad band of wavelengths.

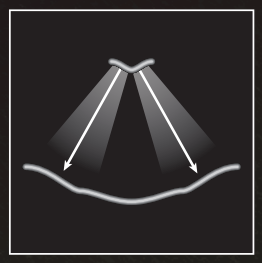

\section{PHASE-INDUCED AMPLITUDE APODIZATION}

Two asymmetrical mirrors reshape the incoming light

beam to shunt starlight off to the side and preserve

planetary light.

Key challenge:

The manufacture of correctly shaped optics.

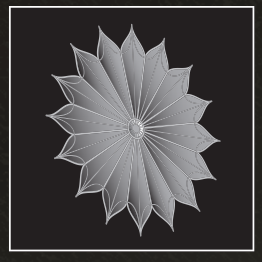

STARSHADE

A petal-shaped structure carried into space on a separate spacecraft shades the telescope optics from the glare of the star.

Key challenge:

Telescope and shade must fly in formation, separated by tens of thousands of kilometres.

\title{
Fresh bid to see exo-Earths
}

\section{Improved instruments and a telescope windfall could aid the search for extrasolar life.}

\section{BY RON COWEN}

A $s$ NASA's Kepler spacecraft finds signs that the Galaxy is teeming with Earthsized planets, astronomers are renewing a stalled quest: to gather light directly from an Earth twin and tease it apart for the chemical signatures of life. That is not possible with current techniques, which find planets indirectly; and in 2006, NASA cancelled a mission that might have had a chance at doing so. The Terrestrial Planet Finder (TPF) would have used either an array of small telescopes or one giant, 8-metre mirror to capture planetary light. Now a new generation of instruments is raising hopes that a smaller, cheaper space telescope could do the job.

The TPF cancellation was "a big blow", says Olivier Guyon, an astronomer at the University of Arizona in Tucson who also works on Japan's Subaru Telescope in Hawaii, "but it also felt like a challenge to see what we could do with smaller telescopes". Guyon and others are hoping to meet that challenge with advanced coronagraphs: telescope devices that mask starlight like an artificial eclipse, allowing nearby planets that would otherwise be obscured by the star's glare to be seen (see 'Blot out the light'). NASA is exploring the idea of putting a coronagraph on one of a pair of 2.4-metre space telescopes donated to the agency by the National Reconnaissance Office, which operates the US fleet

of spy satellites (see Nature 490, 16-17; 2012). Although a telescope that size - the same as the Hubble Space Telescope - would be hardpressed to gather light from Earth-sized planets, it could image and take chemical spectra from planets the size of Jupiter and possibly even smaller than Neptune.

The impetus for such a mission is growing. This month, at a meeting of the American Astronomical Society in

Long Beach, California, astronomers estimated the number of planets in the Milky Way, extrapolated from the number and types of planet that Kepler has already found

\section{"It felt like a challenge to see what we could do with smaller telescopes."}

in one small part of the Galaxy. The result: at least 100 billion extrasolar planets, one for every star. Even more tantalizingly, a potentially habitable Earth-sized orb is likely to reside within 6 parsecs (20 light years) of the Solar System.

Yet NASA, strapped for funds and burdened with the US\$8-billion James Webb Space Telescope project, is not about to resurrect the TPF. Planet hunters know that a smaller, pathfinder mission is their best chance. "This is the only show in town," says Alan Boss, an astronomer at the Carnegie Institution for Science in Washington DC.

A NASA-appointed team of astronomers, the Astrophysics Focused Telescope Assets

committee, will on 30 April deliver a report on the feasibility of using the donated telescopes for several types of observation, including planet imaging. Any planet hunting would have to be squeezed into a jam-packed operations schedule: astrophysicists are also eager to use the telescopes to study the mysterious phenomenon known as dark energy, which is accelerating the expansion of the Universe.

The structure of the instruments could also be a problem. The 2.4-metre telescopes contain two mirrors; the secondary one is held by six struts that cause light scattering, says Wes Traub, chief scientist for NASA's exoplanet exploration programme at the Jet Propulsion Laboratory in Pasadena, California. "The struts couldn't be in a worse place for imaging planets," he says.

Even so, exoplanet astronomers are enthusiastic. Coronagraphs have improved vastly in recent years: just a decade ago, says Guyon, they were good at blotting out most of the star's central light, but also threw away about $90 \%$ of the light reflected from the planet. One problem was caused by the sharp edges of the telescope mirror, which created a ring-like diffraction pattern in the region of the image where the planet resides.

\section{DNATURE.COM}

For more about exoplanets, see: go.nature.com/pxgbbt
Guyon's coronagraph uses two asymmetrically polished mirrors to reshape the distribution of light gathered by the 
telescope's main mirror and eliminate the diffraction pattern. The design, which in 2012 won him a 'genius' grant from the MacArthur Foundation, allows the starlight to be thrown away without sacrificing light from the planet. Guyon says that his device could enable a 4-metre telescope, half the diameter of the TPF, to see an Earth twin - but whether it could enable a 2.4-metre telescope to find Earths remains to be seen. "We're still in the grey zone," he says.
Dust adds to the uncertainty. Asteroid collisions have filled the inner Solar System with a haze of dust that reflects sunlight, creating what is known as zodiacal light. The worst nightmare of planet hunters is the possibility that the dust in other solar systems could generate so much 'exozodiacal' light that Earth-sized orbs would be lost within it. "This is the main non-technological risk for an exo-Earth imaging mission," says Ruslan Belikov, an astrophysicist at the
NASA Ames Research Center in Moffett Field, California. Measuring the levels of exozodiacal light around Sun-like stars is a key goal for a pathfinder mission, he adds.

Even if NASA decides against putting a coronagraph on one of the donated telescopes, says Guyon, he is not about to give up. Finding out if there are other habitable planets in the Galaxy, he says, "is the most interesting question in all of science".

\title{
Japan's stimulus package showers science with cash
}

\author{
But new leadership's largesse brings expectations of fast commercial pay-offs.
}

\section{BY DAVID CYRANOSKI IN TOKYO}

$\mathrm{T}$ Three years ago, the picture for research funding in Japan looked bleak. As part of efforts to slash the national budget by $¥ 3$ trillion (US $\$ 33.5$ billion), the government, led by the Democratic Party of Japan, had proposed sweeping cuts to science, sparking protests from the country's most eminent researchers. Japan's flagship K supercomputer project narrowly escaped being shut down after auditors questioned whether Japan needed to host the world's fastest computer.

Fast-forward to 2013, and Shinzo Abe, head of the newly elected Liberal Democratic Partyled government, seems to have no such doubts. "Of course we must aim for number one," he told reporters after a tour of the supercomputer facility on 11 January.

Science is a big winner in the government's massive $¥ 10.3$-trillion economic stimulus package, approved by the cabinet on 15 January. The stimulus flags up the new leadership's determination that research should pull its weight in dragging Japan's economy out of recession. The supplementary budget provides billions of yen for key fields and significant boosts for many big scientific facilities (see 'Big winners') — including $¥ 8.4$ billion for data links between the K supercomputer and Japan's universities.

Among the many other winners is the SPring-8 synchrotron, which will get $¥ 2.9$ billion to spruce up its ageing beamlines, used for structural studies of materials and proteins. There is also $¥ 16.6$ billion for the international fusion energy project ITER, which has been beset by budgetary difficulties since its inception (see Nature 487, 420; 2012). Meanwhile, the Japanese space agency JAXA will get $¥ 22.9$ billion to refurbish its facilities and to speed up development of ALOS-2, a satellite that will monitor natural disasters and measure atmospheric carbon dioxide levels. The extra cash will keep it on schedule for launch before April next year.

But the biggest beneficiary of the Abe government's stimulus is stem-cell research, especially that geared towards clinical applications. The science ministry alone has earmarked $¥ 21.4$ billion for research on stem cells, mainly focused on induced pluripotent stem (iPS) cells - reprogrammed adult cells, first developed in Japan.

Yoshiki Sasai, who has been wowing biologists and non-scientists alike by growing rudimentary retinas, brain parts and other tissues from stem cells (see Nature 488, 444-446; 2012), has long been negotiating with the government for facilities to link basic research at the Center for Developmental Biology in Kobe, where he works, with clinics and industry. The

\section{BIG WINNERS}

Some of the facilities or fields that receive big funding boosts in Japan's supplementary budget.

\section{IPS CELLS*}

\section{JAXA}

\section{CHIKYU DEEP-SEA DRILLING PROJECT}

SACLA/SPRING-8 $¥$

ITER $\$$

NANOTECH AND MATERIALS

SEA-FLOOR SURVEY

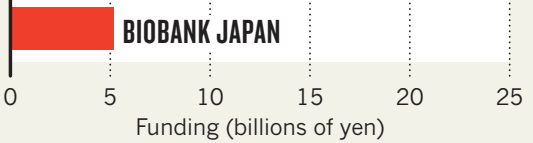
*Induced pluripotent stem cells; †Japanese space agency; ISACLA X-ray
free electron laser/SPring-8 synchrotron; \&lnternational fusion project stimulus package, nonetheless, came "like thunder, out of the blue", he says. The budget includes $¥ 3.8$ billion for a new building at Sasai's centre, with planned projects being largely collaborative ventures with Japanese businesses.

An even bigger windfall will benefit work by Shinya Yamanaka, a Nobel laureate who developed the first recipe for iPS cells. The Center for iPS Cell Research and Application at Kyoto University, which he directs, is to receive $¥ 4$ billion for a building to house research on reprogramming mechanisms and clinical applications of iPS cells. Another $¥ 700$ million will pay to create a cell-processing centre at the Foundation for Biomedical Research and Innovation in Kobe, mainly to support Masayo Takahashi, who works next door at the Center for Developmental Biology. Takahashi is planning the first trial of iPS cells in humans, in a clinical study set to start this year on the eye condition known as macular degeneration.

Other ministries have also jumped on the iPS-cell bandwagon. For example, with $¥ 2.2$ billion the health ministry plans to build two centres to provide training on deriving and cultivating iPS cells.

The stimulus cash could be just the start of the good times for Japan's stem-cell researchers. The government is expected to announce a tenyear, ¥90-billion iPS-cell initiative in next year’s budget, beginning in April.

But the largesse comes with expectations. Of the $¥ 721$ billion earmarked for science and technology by the science ministry, $¥ 180$ billion will go towards translating university research into commercial applications, and most other projects are framed around clinical or industrial applications. With the stimulus, says Yamanaka, the government is "telling us to rush iPS-cell-related technologies to patients as quickly as possible". . 